\title{
ARTROPLASTIA PARCIAL NO TRATAMENTO DAS FRATURAS DO COLO DO FÊMUR
}

\section{HEMIARTHROPLASTY IN THE TREATMENT OF FRACTURES OF THE FEMORAL NECK}

\begin{abstract}
Nelson Keiske Ono', Guilherme Didier de Andrade Lima², Emerson Kiyoshi Honda ${ }^{3}$, Giancarlo Cavalli Polesello4, Rodrigo Pereira Guimarães ${ }^{5}$, Walter Ricioli Júnior ${ }^{6}$, Marcelo Cavalheiro de Queiroz ${ }^{6}$
\end{abstract}

\section{RESUMO}

Objetivo: Realizar avaliação epidemiológica e clínica dos pacientes com fratura desviada do colo femoral, que foram submetidos ao tratamento cirúrgico, com artroplastia parcial do quadril cimentada. Métodos: Foram avaliados, de forma retrospectiva, todos os pacientes com fratura desviada do colo do fêmur (Garden III e IV) submetidos à artroplastia parcial do quadril com prótese unipolar (Thompson), cimentada pela via de acesso posterolateral do quadril, no período de junho de 2005 a setembro de 2008. Resultados: Foram avaliados, inicialmente, 70 pacientes. A média de idade foi de 83,1 anos. Houve predomínio de pacientes do sexo feminino (84,3\%). Houve acompanhamento ambulatorial de 36 pacientes, cujo tempo de seguimento variou de 10 a 48 meses (média de 26,5 meses). Houve perda de seguimento de 15 pacientes. Dezenove pacientes foram a óbito, com uma taxa de mortalidade no primeiro ano de 25,4\%. Os pacientes classificados como ASA III apresentaram taxa de 25,7\%, enquanto os pacientes ASA II, uma taxa de $12,1 \%$. Dois pacientes apresentaram trombose venosa profunda sintomática; um paciente, infecção do sítio operatório; e nenhum paciente apresentou luxação do quadril. A maioria dos pacientes evoluiu sem dor. Doze pacientes (33\%), durante a evolução, apresentaram piora na capacidade de deambulação. Conclusão: Nenhum caso de luxação do quadril foi observado. Os pacientes classificados como ASA III apresentaram um índice de mortalidade mais elevado, em relação aos pacientes ASA I e II. Houve uma piora da capacidade de deambular em 33\% dos pacientes. Não foi necessária revisão de nenhum paciente por soltura ou dor. Trinta pacientes não apresentavam dor (83,3\%), quatro apresentavam dor moderada $(11,1 \%)$ e dois apresentavam dor intensa (5,5\%).

Descritores - Fraturas do colo femoral; Idoso; Artroplastia de quadril

\section{ABSTRACT}

Objective: To epidemiologically and clinically evaluate patients with displaced femoral neck fractures that had surgical treatment with cemented hemiarthroplasty. Methods: We evaluated, retrospectively, all patients with displaced femoral neck fractures (Garden III and IV) that had surgical treatment with cemented partial hip replacement (Thompson), using the posterior approach, from June 2005 to September 2008. Results: We initially evaluated 70 patients. The mean age was 83.1 years. Most of the patients were female (84.3\%). Thirty-six patients were monitored for a period ranging from 10 to 48 months (mean follow-up period of 26.5 months). Fifteen patients were lost to follow-up. Nineteen patients died within the first year, with a mortality rate of 25.4\%. Patients classified as ASA III had a mortality rate of $25.7 \%$ and ASA II patients, a $12.1 \%$ rate. Two patients had symptomatic deep vein thrombosis; one patient had a superficial wound infection; no patient had dislocation. Most of the patients did not experience pain. Twelve patients (33\%) showed deterioration in their walking ability. Conclusion: There was no hip dislocation. Patients classified as ASA III had a higher death rate than ASA I or II. There was a worsening of walking ability in 33\% of the patients. No revision was necessary due to loosening or pain. Thirty patients didn't present pain (83.3\%), four presented with moderate pain (11.1\%), and two presented with intense pain (5.5\%).

Keywords - Femoral neck fractures; Aged; Arthroplasty, Replacement hip

\footnotetext{
1 - Professor Assistente, Doutor da FCMSCSP e Chefe do Grupo de Quadril - ISCMSP.

2 - Médico Ortopedista, Estagiário do Grupo de Quadril - ISCMSP.

3 - Professor Assistente, Doutor da FCMSCSP e Membro Sênior do Grupo de Quadril - ISCMSP.

4 - Professor Assistente, Doutor da FCMSCSP e Membro do Grupo de Quadril - ISCMSP.

5 - Professor Instrutor, Mestre da FCMSCSP e Membro do Grupo de Quadril - ISCMSP.

6 - Médico Ortopedista, Membro do Grupo de Quadril - ISCMSP.
}

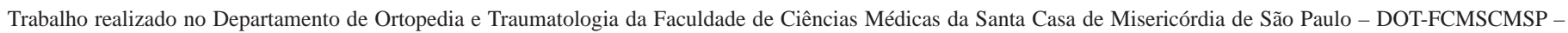
Diretor: Prof. Dr. Osmar Avanzi - São Paulo (SP), Brasil

Correspondência: Pavilhão “Fernandinho Simonsen”, Rua Dr. Cesário Motta Jr., 112 - 01221-020 - São Paulo (SP) - Brasil. E-mail: nko@uol.com.br 


\section{INTRODUÇÃO}

As fraturas do colo femoral são intracapsulares e tipicamente ocorrem em uma distribuição etária bimodal, sendo a maioria na população idosa ${ }^{(1)}$. As opções de tratamento, incluem a abordagem não-cirúrgica, a fixação percutânea, a redução aberta e fixação interna e artroplastia (parcial ou total) do quadril ${ }^{(1)}$.

As decisões do tratamento das fraturas do colo femoral são, comumente, baseadas em dois aspectos. O primeiro aspecto é a condição clínica do paciente, que inclui a idade, nível de atividade e comorbidades. O segundo aspecto é o tipo de fratura, mais especificamente, se desviada ou não-desviada. O paciente, idoso fisiologicamente, com uma fratura do colo femoral deslocada, que tem baixa demanda, tem sido tratado com a prótese parcial de Thompson.

Este estudo tem o objetivo de realizar uma avaliação epidemiológica e clínica, dos pacientes com fratura desviada do colo femoral, que foram submetidos ao tratamento cirúrgico com artroplastia parcial do quadril unipolar (Thompson) cimentada.

\section{CASUÍSTICA E MÉTODOS}

Foram avaliados todos os pacientes com fratura do colo do fêmur deslocada (Garden III e IV), que foram submetidos à artroplastia parcial do quadril com prótese unipolar Thompson, cimentada pela via de acesso posterolateral do quadril. Todos os pacientes foram operados no Departamento de Ortopedia e Traumatologia da Faculdade de Ciências Médicas da Santa Casa de Misericórdia de São Paulo, no período de junho de 2005 a setembro de 2008.

Os pacientes foram submetidos, na admissão hospitalar, a radiografias nas incidências anteroposterior da pelve e perfil do quadril, sendo as fraturas classificadas de acordo com Garden ${ }^{(2)}$. E, após radiografias e avaliação clínica, foi preenchido o protocolo elaborado pelo Grupo de Afecções do Quadril do Departamento de Ortopedia e Traumatologia da Faculdade de Ciências Médicas da Santa Casa de Misericórdia de São Paulo.

Foram admitidos 70 pacientes com fratura do colo do fêmur desviada, sendo todos submetidos a tratamento cirúrgico com artroplastia parcial do quadril, com prótese de Thompson cimentada pela via de acesso posterolateral.

Quanto à técnica operatória, o paciente é posicionado em decúbito lateral. Realiza-se via de acesso posterolateral de Moore ${ }^{(3)}$; resseca-se a cabeça femoral e o colo femoral; fresa-se o canal femoral; faz-se a oclusão do canal femoral com rolha óssea; realiza-se a cimentação da prótese unipolar de Thompson, com 10 a $15^{\circ}$ de anteversão; testa-se a estabilidade do quadril com o membro inferior em flexão, rotação interna e adução, e em extensão e rotação externa; e realiza-se a sutura da cápsula posterior e reinserção, quando possível, dos músculos rotadores externos curtos, com pontos transósseos com fio de Ethibond ${ }^{\circledR}$.

Foi adotada a profilaxia mecânica para trombose venosa profunda no pós-operatório imediato, com fisioterapia motora e mobilidade precoce do paciente. Não foi realizada a profilaxia medicamentosa.

$\mathrm{Na}$ avaliação da capacidade de marcha, os pacientes foram classificados em quatro grupos: deambulador comunitário (paciente com capacidade de deambular dentro e fora da residência, podendo necessitar de auxílio à marcha); deambulador domiciliar (paciente com capacidade de deambular apenas na residência, geralmente necessitando auxílio para marcha); deambulador nãofuncional (paciente que deambula apenas nas sessões de fisioterapia); e não-deambulador (sem capacidade para deambular) ${ }^{(4)}$.

Com relação à dor, foi utilizada a escala de dor de Sikorski e Barrington ${ }^{(5)}$. Essa escala foi adotada por ser de fácil aplicação e podendo ser respondida pelo paciente e por seus acompanhantes, pois muitos pacientes apresentaram alterações cognitivas importantes, não sendo capazes de responder a determinadas perguntas e quantificar a dor. Essa escala avalia a presença de dor e se há necessidade e frequência do uso de analgésicos (Quadro 1).

Quadro 1 - Escala para dor de Sikorski e Barrington ${ }^{(5)}$

\begin{tabular}{|l|l|}
\hline Grau 1 & dor ausente \\
\hline Grau 2 & dor moderada, ocasional, não requerendo analgésicos \\
\hline Grau 3 & dor constante ou ocasional e intensa, requerendo analgésicos ocasionais \\
\hline Grau 4 & dor constante e intensa, requerendo regularmente analgésicos \\
\hline
\end{tabular}

Todos os pacientes foram encaminhados ao ambulatório do Grupo do Quadril para acompanhamento periódico, sendo obtidas radiografias na incidência anteroposterior da pelve. Foram preenchidos os protocolos de acompanhamento ambulatorial, elaborados pelo Grupo. Entre vários aspectos analisados, avaliou-se a presença de infecção (clínica e laboratorialmente), trombose venosa profunda e luxação da prótese. 


\section{RESULTADOS}

Dos 70 pacientes: 59 sexo feminino (84,3\%) e 11 sexo masculino (15,7\%). Trinta e sete pacientes tiveram o lado direito acometido (52,9\%). A média de idade foi de 83,1 anos, variando de 58 a 99 anos. Pela classificação da American Society of Anesthesiology (ASA), dois pacientes eram ASA I (2,86\%), 33 ASA II (47,14\%) e 35 ASA III (50\%). Nenhum paciente foi classificado como ASA IV ou V. Todas as fraturas do colo do fêmur eram desviadas, sendo 20 (28,6\%) do grau III de Garden e 50 (71,4\%) grau IV. Todos os pacientes apresentaram trauma de baixa energia, com queda ao solo da própria altura.

O tempo médio entre a internação e o procedimento cirúrgico foi de 7,86 dias, variando de menos de 24 horas a 40 dias.

Dos 70 pacientes admitidos inicialmente, houve perda de seguimento de 15 pacientes, e 19 óbitos. Houve acompanhamento ambulatorial de 36 pacientes. $\mathrm{O}$ tempo de seguimento variou de 10 meses a 48 meses, com média de 26,5 meses.

Observou-se óbito de 19 pacientes. Desses óbitos, $14(25,4 \%)$ foram no primeiro ano após o procedimento cirúrgico, sendo oito no primeiro trimestre, quatro no segundo e dois no terceiro trimestre.

Dos pacientes que foram a óbito, um paciente apresentou TVP no oitavo dia de pós-operatório. Apresentou episódio de TVP um ano antes do procedimento cirúrgico, e ainda estava em uso de Marevan ${ }^{\circledR}$, que foi suspenso para o procedimento cirúrgico. Faleceu no quinto mês de pós-operatório.

Um paciente faleceu durante o procedimento cirúrgico. Após a cimentação do canal femoral, o paciente apresentou hipotensão e parada cardiorrespiratória, não respondendo aos procedimentos de reanimação.

As causas dos demais 17 óbitos não foram relacionadas diretamente com o procedimento cirúrgico. Nenhum desses pacientes apresentou infecção do sítio operatório, sintomas de trombose venosa profunda e luxação do quadril.

Dos 36 pacientes acompanhados no ambulatório, nenhum apresentou luxação do quadril.

Um paciente apresentou infecção aguda no sítio operatório, sendo submetido à limpeza cirúrgica e antibioticoterapia sem sucesso, necessitando de artroplastia de ressecção (Girdlestone) após 10 meses do procedimento cirúrgico inicial.

Um paciente apresentou sinais clínicos de trombose venosa profunda no acompanhamento ambulatorial. Tal paciente foi submetido à ultrassonografia Doppler do membro inferior acometido, o qual evidenciou trombose venosa profunda. O paciente foi submetido ao tratamento com anticoagulante oral, em acompanhamento conjunto com a cirurgia vascular.

Nenhum paciente apresentou necessidade de revisão por dor ou soltura da prótese.

Quando se avaliou a classificação ASA com o tempo de internação até a cirurgia, observamos que os pacientes ASA III necessitaram de um tempo maior para compensação clínica (Tabela 1).

Tabela 1 - Relação entre a Classificação ASA e o tempo entre a internação e a cirurgia

\begin{tabular}{l|l}
\hline ASA I & 3,5 dias ( 1 e 7 dias) \\
\hline ASA II & 6,6 dias (1 a 17 dias) \\
\hline ASA III & 9,3 dias (menos 24h a 40 dias) \\
\hline
\end{tabular}

Com relação à capacidade de marcha, os pacientes foram, no período pré-operatório, classificados em: 25 deambuladores comunitários, 10 deambuladores domiciliares e um não-deambulador. Ao final do período de acompanhamento deste estudo, observou-se que 24 pacientes (66\%) mantiveram a mesma capacidade de marcha. Dos pacientes que apresentaram queda do padrão, dois deambuladores domiciliares passaram a nãodeambular, sete deambuladores comunitários passaram a ser deambuladores comunitários, e três deambuladores comunitários tornaram-se não-deambuladores. Não houve nenhum caso de melhora da capacidade de marcha.

Quando avaliada a presença da dor, 30 pacientes referiram não sentir dor; quatro, referiram dor de intensidade moderada, mas que não necessitava de analgésicos, e dois pacientes referiram dor intensa que necessita de analgésicos ocasionais. Nenhum paciente referiu dor intensa e que necessitava de analgésicos regularmente (Tabela 2).

Tabela 2 - Escala para dor de Sikorski e Barrington ${ }^{(5)}$

\begin{tabular}{c|l|c|c}
\hline Grau 1 & dor ausente & 30 & $83,3 \%$ \\
\hline Grau 2 & $\begin{array}{l}\text { dor moderada, ocasional, não requerendo } \\
\text { analgésicos }\end{array}$ & 4 & $11,1 \%$ \\
\hline Grau 3 & $\begin{array}{l}\text { dor constante ou ocasional e intensa, requerendo } \\
\text { analgésicos ocasionais }\end{array}$ & 2 & $5,6 \%$ \\
\hline Grau 4 & $\begin{array}{l}\text { dor constante e intensa, requerendo regularmente } \\
\text { analgésicos }\end{array}$ & 0 & 0 \\
\hline & & 36 & $100 \%$ \\
\hline
\end{tabular}




\section{DISCUSSÃO}

Em estudo realizado na cidade de São Paulo, Chikude et $\mathrm{l}^{(6)}$ observaram, nos pacientes com fratura do colo do fêmur que foram submetidas a artroplastia parcial do quadril, uma predominância do sexo feminino (80\%) e uma média de idade de 83 anos (variando de 70 a 95 anos). O presente estudo confirma esses dados, com uma prevalência do sexo feminino de $84,3 \%$ e uma média de idade de 83,1 anos.

A classificação radiográfica mais utilizada para as fraturas do colo do fêmur é a de Garden. Estudos mostram que é alta a variação intra e interobservadores quando se utiliza esta classificação. A classificação de Garden é apurada para dividir as fraturas em não-deslocada (Garden I e II) e deslocada (Garden III e IV) ${ }^{(7-9)}$. Os pacientes que foram submetidos à artroplastia parcial foram os que apresentaram fraturas deslocadas.

A American Society of Anesthesiology possui um sistema de classificação quanto ao risco anestésico. Nesse sistema, o grau I indica paciente saudável; o grau II, doença sistêmica leve, sem limitação funcional; o grau III, doença sistêmica moderada, com limitação funcional; o grau IV, doença sistêmica severa que representa risco de vida constante; o grau $\mathrm{V}$, paciente moribundo com perspectiva de óbito dentro de 24 horas, com ou sem cirurgia ${ }^{(10)}$. White et al ${ }^{(11)}$ consideraram a classificação ASA como o melhor preditor de mortalidade para pacientes com fratura do quadril. No seu estudo, os pacientes classificados como grau I e II apresentaram uma taxa de mortalidade, após um ano, de $8 \%$, enquanto os pacientes com grau III e IV apresentaram uma taxa de 49\%. No presente estudo, dos 35 pacientes classificados como ASA III, nove $(25,7 \%)$ foram a óbito no primeiro ano após o procedimento cirúrgico. Os ASA II eram 33, com quatro $(12,1 \%)$ falecendo no primeiro ano.

As próteses utilizadas na artroplastia parcial podem ser agrupas em dois grupos: bipolar ou unipolar. Existe na literatura um grande debate sobre as vantagens e desvantagens de cada grupo. Vários estudos, analisando os dois grupos, concluíram que os resultados são semelhantes no que se refere ao desgaste acetabular, resultados funcionais (capacidade de deambular, realizar atividades da vida diária), duração da cirurgia, perda sanguínea, infecção da ferida, qualidade de vida, dor no quadril ou mortalidade. Além disso, o custo da prótese unipolar é, aproximadamente, $25 \%$ da prótese bipolar ${ }^{(12-15)}$. No presente estudo, todos os pacientes foram submetidos à artroplastia parcial com a prótese unipolar (Thompson).
As artroplastias parciais podem, também, ser agrupadas em outros dois grupos: cimentadas ou não-cimentadas. Estudos mostram que a artroplastia parcial cimentada apresenta incidência menor de dor residual e fratura periprotética, melhor mobilidade, maior independência dos dispositivos de auxílio à marcha, melhor retorno às atividades da vida diária e menor taxa de erosão acetabular. Os argumentos a favor das não-cimentadas são o menor custo, redução do tempo cirúrgico, menor perda sanguínea e menor índice de complicações clínicas relacionadas a não utilização do cimento. Outros estudos mostram que não há diferença em relação a complicações clínicas e índice de mortalidade, entre os pacientes que utilizaram próteses cimentadas e não-cimentadas ${ }^{(1,12,16-19)}$. Em acordo com a grande parte da literatura, todas as artroplastias parciais foram cimentadas.

Lennox e McLaughlan ${ }^{(20)}$ observaram que a artroplastia parcial cimentada apresenta mortalidade transoperatória mais elevada, porém, maior satisfação do paciente, em relação à não-cimentada. As taxas de mortalidade a longo prazo e de complicações foram similares aos dois grupos. No presente estudo, observou-se que houve uma morte no transoperatório, logo após a cimentação do canal femoral.

O índice de mortalidade dos pacientes idosos, com fratura do colo do fêmur, em um ano é elevado, sendo relato desde $18 \%$ a $30 \%$ em pacientes sem comorbidades agudas ${ }^{(18,21,22)}$. Muitos estudos mostram que alguns fatores aumentam a taxa de mortalidade, entre os quais se destacam o sexo masculino, idade avançada, doenças sistêmicas não-controladas, pacientes institucionalizados, pacientes com doenças psiquiátricas, atraso na realização do procedimento cirúrgico e complicações no pós-operatório ${ }^{(23-25)}$.

Muitos estudos mostram que o atraso na realização do procedimento cirúrgico é um dos principais fatores relacionados ao aumento da taxa de mortalidade. Entretanto, não existe um tempo definido limítrofe. Faz-se necessário, num primeiro momento, avaliar se o paciente apresenta alguma comorbidade médica aguda (por exemplo, insuficiência cardíaca, anemia, arritmia cardíaca, distúrbio hidroeletrolítico, pneumonia, distúrbios na coagulação, entre outras) ou não. Para os pacientes sem comorbidades agudas, os estudos clínicos indicam que a cirurgia deve ser realizada dentro das primeiras 24-48h, a até quatro dias após o trauma, com o objetivo de diminuir os riscos de complicações ${ }^{(7,22,26)}$. 
Já os pacientes com fratura do colo femoral e comorbidades médicas agudas são verdadeiros desafios para a equipe médica. Esses pacientes têm um risco de mortalidade elevado, com taxas de 17\% em 30 dias, e $43 \%$ em um ano. Em geral, a cirurgia deveria ocorrer quando as condições do paciente são adequadas ao procedimento; entretanto, essa é uma decisão difícil, que necessita ser individualizada e deveria envolver anestesista, clínico geral e cirurgião. Para esse paciente não há um tempo ideal para o procedimento cirúrgico, mas o retardo da cirurgia é associado com o aumento da mortalidade ${ }^{(22)}$.

Confrontando esses estudos, Kenzora et al afirmam que o atraso para realizar a cirurgia estava associado a um decréscimo na taxa de mortalidade. Foi relatada uma taxa de $28 \%$ para pacientes saudáveis (aqueles que tinham três ou menos comorbidades), que foram submetidos ao procedimento cirúrgico no dia da admissão, e uma taxa de $4 \%$ para os que foram submetidos entre o segundo e o quinto dia de admissão. Concluíram que, todos os pacientes que apresentaram fratura do colo do fêmur necessitam de um a dois dias para se recuperarem das alterações fisiológicas agudas, induzidas pela fratura. São realizadas algumas críticas a este estudo. O grupo estudado foi heterogêneo, incluindo pacientes sem alterações cognitivas, com capacidade de deambular, até pacientes institucionalizados, não-deambuladores e com demência, o que pode obscurecer o impacto da cirurgia, retardada na sobrevida dos pacientes que são relativamente saudáveis antes da fratura ${ }^{(27)}$.

Sexson e Lehner ${ }^{(23)}$, em um estudo retrospectivo, observaram que pacientes que possuíam duas ou menos comorbidades apresentaram uma taxa elevada de mortalidade em um ano (15\%), quando a cirurgia era adiada por mais de 24 horas em relação aos pacientes que foram submetidos à cirurgia dentro das primeiras 24 horas (3\%). Em contraste, os pacientes que possuíam três ou mais comorbidades tiveram uma taxa de mortalidade de um ano menor, quando a cirurgia foi adiada (22\%) em relação aos pacientes em que a cirurgia não foi adiada (33\%).

No presente estudo, o tempo médio entre a internação e o procedimento cirúrgico foi de 7,86 dias. Apenas oito pacientes foram operados nas primeiras 48 horas após a internação, sendo um ASA I, quatro ASA II e três ASA III. Todos os três pacientes ASA III e o paciente ASA I foram a óbito no período pós-operatório. Houve seguimento de três pacientes ASA II, onde não foi observado nenhum óbito. Há perda de seguimento de um paciente ASA II. Houve a necessidade em adiar o procedimento cirúrgico, na grande maioria dos casos, para compensação clínica dos pacientes, sendo sempre realizada avaliação conjunta da equipe médica (ortopedista, clínico geral e anestesiologista).

A via de acesso na artroplastia parcial do quadril é motivo de controvérsia na literatura. Estudos mostram que a via de acesso afeta a incidência de luxações, infecções, duração da cirurgia e perda sanguínea. As vias mais utilizadas são a anterolateral e a posterolateral ${ }^{(1)}$. Estudos relatam que a via de acesso posterolateral é associada a uma maior taxa de luxação do quadril, apresentando taxas de até $16,3 \%$, contra uma taxa de 3,3\% na via de acesso anterolateral. Devido a essas taxas elevadas, muitos estudos sugerem que a via de acesso posterolateral deveria ser abandonada na artroplastia parcial do quadril ${ }^{(28,29)}$, pois a luxação do quadril já é considerada, pela literatura, uma verdadeira catástrofe, com taxas médias de mortalidade relatas entre $50 \%$ a $65 \%$, chegando até a impressionante taxa de $73 \%$ em seis meses ${ }^{(30-33)}$. Entretanto, outros estudos demonstraram que não houve diferença nas taxas de luxação, após artroplastia parcial do quadril, com relação à via de acesso posterolateral e anterolateral ${ }^{(5,34)}$. Ko et al ${ }^{(35)}$ recomendam o reparo da cápsula articular e reinserção dos músculos rotadores externos curtos na via de acesso posterior, pois não encontraram nenhum caso de luxação do quadril, após artroplastia parcial do quadril, com a reparação, e uma taxa de 1,9\% quando não foi realizado o reparo posterior. No presente estudo, a via de acesso utilizada foi a posterolateral, e o reparo dos músculos rotadores externos curtos foi realizada, quando possível. Não foi observado nenhum caso de luxação do quadril.

A taxa de trombose venosa profunda é elevada na via de acesso posterior, com taxas relatadas de até 6,5\% (8). E a trombose venosa profunda subclínica certamente é bem mais elevada (8). No presente estudo, a taxa de TVP foi de 3,6\%. Foi utilizada a profilaxia mecânica, com mobilização precoce (ativa e passiva) e fisioterapia motora. Dos dois pacientes que apresentaram sinais clínicos de TVP, um paciente tinha história prévia de TVP e ainda estava em tratamento com Marevan ${ }^{\circledR}$, que evoluiu para óbito. E o outro paciente não tinha história de TVP e nem fatores de risco, evoluindo satisfatoriamente com o tratamento clínico com anticoagulantes orais.

A taxa de infecção profunda em pacientes idosos submetidos à artroplastia parcial do quadril com prótese 
unipolar, utilizando a via de acesso posterior é descrita variando de $1,5 \%^{(33)}$ a $4 \%{ }^{(5)}$. A taxa de infecção profunda encontrada neste estudo foi de 1,8\%. O paciente foi submetido à limpeza cirúrgica e antibioticoterapia endovenosa, sem sucesso terapêutico, evoluindo com persistência do quadro clínico. Foi submetido, então, à artroplastia de ressecção do quadril infectado, evoluindo com cura do quadro infeccioso.

A dor após a cirurgia não é intensa, na maior parte dos casos e não limita o indivíduo em suas atividades. Vilas Boas Junior et $a^{(36)}$ avaliando pacientes com fratura do colo do fêmur, submetidos a tratamento cirúrgico com hemiartroplastia ou fixação interna, observaram que $6,45 \%$ dos pacientes se queixavam de dor constante e intensa, requerendo a administração de analgésicos, enquanto que $93,55 \%$ dos pacientes não tinham dor ou esta era ocasional e leve. No presente estudo, dois pacientes (5,55\%) apresentaram dor intensa, ocasionalmente necessitando de analgésicos, enquanto 94,45\% não apresentavam dor ou esta era moderada, não requerendo analgésicos.

Um dos principais objetivos do procedimento cirúrgico é que o paciente retorne, pelo menos, ao nível de marcha que apresentava antes da fratura. Na literatura, os índices dos pacientes que recuperam a capacidade de deambular variam de $41 \%$ a $97 \%$. Tal variação, provavelmente, ocorre pela falta de padronização dos métodos que avaliam a marcha dos pacientes ${ }^{(25)}$. Miller relatou que $22 \%$ dos pacientes perderam a capacidade de deambular ${ }^{(24)}$. Os pacientes que são ativos e independentes no período pré-trauma são os que apresentam os menores índices de limitação funcional após a cirurgia Os fatores que mais se correlacionam com os maus resultados funcionais são a debilidade muscular e a dificuldade de equilíbrio dos pacientes ${ }^{(36)}$. Cunha e Veado, analisando pacientes com fratura do fêmur proximal, observaram que 1/3 dos pacientes apresentam algum grau de dependência funcional ${ }^{(37)}$. Observamos que 12 pacientes (33\%) apresentaram queda no padrão da marcha. A queda mais significativa foi observada nos deambuladores comunitários, pois 11 pacientes (44\% dos deambuladores comunitários) apresentaram piora, sendo que sete tornaram-se deambuladores domiciliares e quatro, não-deambulares. E apenas um paciente deambulador domiciliar apresentou piora, tornando-se não-deambulador.

Devido ao curto follow up e a grande perda de pacientes, não localizados e falecidos (cerca de 50\%), não foi possível fazer uma avaliação adequada da prótese de Thompson cimentada.

\section{CONCLUSÃO}

Houve uma predominância do sexo feminino. Todos os pacientes sofreram trauma de baixa energia.

O tempo médio entre internação e procedimento cirúrgico foi de 7,86 dias.

Foi encontrado alto índice de mortalidade no pósoperatório, com taxa de mortalidade no primeiro ano de 25,4\%. Os pacientes classificados como ASA III apresentaram um índice de mortalidade mais elevado em relação aos pacientes ASA I e II.

Não foi observado nenhum caso de luxação.

Foi observada piora da capacidade de deambulação em 33\% dos casos.

Não houve necessidade de revisão de nenhum paciente por soltura ou dor.

Trinta pacientes não apresentavam dor (83,3\%), quatro apresentavam dor moderada $(11,1 \%)$ e dois apresentavam dor intensa (5,5\%).

\section{REFERÊNCIAS}

1. Miyamoto RG, Kaplan KM, Levine BR, Egol KA, Zuckerman JD. Surgical management of hip fractures: an evidence-based review of the literature. I: femoral neck fractures. J Am Acad Orthop Surg. 2008;16(10):596-607.

2. Garden RS. Low-angle fixation in fractures of the femoral neck. J Bone Joint Surg Br. 1961;43:647-63.

3. Moore AT. The self-locking metal hip prosthesis. J Bone Joint Surg Am. 1957;39(4):811-27.

4. Hoffer MM, Feiwell E, Perry R, Perry J, Bonnett C. Functional ambulation in patients with myelomeningocele. J Bone Joint Surg Am. 1973;55(1):137-48.

5. Sikorski JM, Barrington R. Internal fixation versus hemiarthroplasty for the displaced subcapital fracture of the femur. A prospective randomised study. J Bone Joint Surg Br. 1981;63(3):357-61.

6. Chikude T, Fujiki EN, Honda EK, Ono NK, Milani C. Avaliação da qualidade de vida dos pacientes idosos com fratura do colo do fêmur tratados cirurgicamente pela artroplastia parcial do quadril. Acta Ortop Bras. 2007;15(4):197-9.

7. Parker MJ. The management of intracapsular fractures of the proximal femur. J Bone Joint Surg Br. 2000;82(7):937-41.

8. Gusmão PDF, Mothes FC, Rubin LA, Gonçalves RZ, Telöken MA, Schwartsmann CR. Avaliação da reprodutibilidade da classificação de Garden para fraturas do colo femoral. Rev Bras Ortop. 2002;37(9):381-6.

9. Thomsen NO, Jensen CM, Skovgaard N, Pedersen MS, Pallesen P, Soe-Nielsen $\mathrm{NH}$, et al. Observer variation in the radiographic classification of fractures of the neck of the femur using Garden's system. Int Orthop. 1996;20(5):326-9.

10. Morgan GE Jr, Mikhail MS. The practice of anesthesiology. Clinical anesthesiology. 2nd ed. Stanford: Appleson \& Lange. 1996. p.1-12.

11. White BL, Fisher WD, Laurin CA. Rate of mortality for elderly patients after fracture of the hip in the 1980's. J Bone Joint Surg Am. 1987;69(9):1335-40.

12. Parker MJ, Gurusamy K. Arthroplasties (with and without bone cement) for proximal femoral fractures in adults. Cochrane Database Syst Rev. 2004;(2):CD001706. 
13. Raia FJ, Chapman CB, Herrera MF, Schweppe MW, Michelsen CB, Rosenwasser MP. Unipolar or bipolar hemiarthroplasty for femoral neck fractures in the elderly? Clin Orthop Relat Res. 2003;(414):259-65.

14. Calder SJ, Anderson GH, Jagger C, Harper WM, Gregg PJ. Unipolar or bipolar prosthesis for displaced intracapsular hip fracture in octogenarians: a randomised prospective study. J Bone Joint Surg Br. 1996;78(3):391-4.

15. Ong BC, Maurer SG, Aharonoff GB, Zuckerman JD, Koval KJ. Unipolar versus bipolar hemiarthroplasty: functional outcome after femoral neck fracture at a minimum of thirty-six months of follow-up. J Orthop Trauma. 2002;16(5):317-22.

16. Emery RJ, Broughton NS, Desai K, Bulstrode CJ, Thomas TL. Bipolar hemiarthroplasty for subcapital fracture of the femoral neck. A prospective randomised trial of cemented Thompson and uncemented Moore stems. J Bone Joint Surg Br. 1991;73(2):322-4

17. Singh GK, Deshmukh RG. Uncemented Austin-Moore and cemented Thompson unipolar hemiarthroplasty for displaced fracture neck of femur--comparison of complications and patient satisfaction. Injury. 2006;37(2):169-74

18. Faraj AA, Branfoot T. Cemented versus uncemented Thompson's prostheses: a functional outcome study. Injury. 1999;30(10):671-5.

19. Khan RJ, MacDowell A, Crossman P, Keene GS. Cemented or uncemented hemiarthroplasty for displaced intracapsular fractures of the hip--a systematic review. Injury. 2002;33(1):13-7.

20. Lennox IA, McLauchlan J. Comparing the mortality and morbidity of cemented and uncemented hemiarthroplasties. Injury. 1993;24(3):185-6.

21. D'Arcy J, Devas M. Treatment of fractures of the femoral neck by replacement with the Thompson prosthesis. J Bone Joint Surg Br. 1976;58(3):279-86.

22. Moran CG, Wenn RT, Sikand M, Taylor AM. Early mortality after hip fracture: is delay before surgery important? J Bone Joint Surg Am. 2005;87(3):483-9.

23. Sexson SB, Lehner JT. Factors affecting hip fracture mortality. J Orthop Trauma. 1987;1(4):298-305

24. Miller CW. Survival and ambulation following hip fracture. J Bone Joint Surg Am. 1978;60(7):930-4.

25. Koval KJ, Zuckerman JD. Functional recovery after fracture of the hip. J Bone
Joint Surg Am. 1994;76(5):751-8.

26. Zuckerman JD, Skovron ML, Koval KJ, Aharonoff G, Frankel VH. Postoperative complications and mortality associated with operative delay in older patients who have a fracture of the hip. J Bone Joint Surg Am. 1995;77(10):1551-6.

27. Kenzora JE, McCarthy RE, Lowell JD, Sledge CB. Hip fracture mortality. Relation to age, treatment, preoperative illness, time of surgery, and complications. Clin Orthop Relat Res. 1984;(186):45-56.

28. Pajarinen J, Savolainen V, Tulikoura I, Lindahl J, Hirvensalo E. Factors predisposing to dislocation of the Thompson hemiarthroplasty: 22 dislocations in 338 patients. Acta Orthop Scand. 2003;74(1):45-8.

29. Unwin AJ, Thomas M. Dislocation after hemiarthroplasty of the hip: a comparison of the dislocation rate after posterior and lateral approaches to the hip. Ann R Coll Surg Engl. 1994;76(5):327-9.

30. Paton RW, Hirst P. Hemiarthroplasty of the hip and dislocation. Injury. 1989;20(3):167-9.

31. Blewitt N, Mortimore S. Outcome of dislocation after hemiarthroplasty for fractured neck of the femur. Injury. 1992;23(5):320-2.

32. Keene GS, Parker MJ. Hemiarthroplasty of the hip--the anterior or posterior approach? A comparison of surgical approaches. Injury. 1993;24(9):611-3.

33. Chan RN, Hoskinson J. Thompson prosthesis for fractured neck of femur. A comparison of surgical approaches. J Bone Joint Surg Br. 1975;57(4):437-43.

34. Sierra RJ, Schleck CD, Cabanela ME. Dislocation of bipolar hemiarthroplasty: rate, contributing factors, and outcome. Clin Orthop Relat Res. 2006;442:230-8.

35. Ko CK, Law SW, Chiu KH. Enhanced soft tissue repair using locking loop stitch after posterior approach for hip hemiarthroplasty. J Arthroplasty. 2001;16(2):207-11.

36. Vilas Boas Junior A, Soni J, Fratti SR, Kantovitz PCJ, Souza Filho RM, Velente Netto EB. A fratura do colo do fêmur como fator de maior morbidade e mortalidade. Rev Bras Ortop. 1998;33(6):483-8.

37. Cunha $U$, Veado MAC. Fratura da extremidade proximal do fêmur em idosos: independência funcional e mortalidade em um ano. Rev Bras Ortop. 2006; 41(6):195-9. 